\title{
Ion cyclotron waves during the Rosetta approach phase: a magnetic estimate of cometary outgassing
}

\author{
M. Volwerk ${ }^{1}$, C. Koenders ${ }^{2}$, M. Delva ${ }^{1}$, I. Richter ${ }^{2}$, K. Schwingenschuh ${ }^{1}$, M. S. Bentley ${ }^{1}$, and K.-H. Glassmeier ${ }^{2}$ \\ ${ }^{1}$ Space Research Institute, Austrian Academy of Sciences, 8042 Graz, Austria \\ ${ }^{2}$ Institute for Geophysics and Extraterrestrial Physics, TU Braunschweig, Germany \\ Correspondence to: M. Volwerk (martin.volwerk@oeaw.ac.at)
}

Received: 21 October 2013 - Revised: 21 November 2013 - Accepted: 21 November 2013 - Published: 17 December 2013

\begin{abstract}
A theoretical model for the ion cyclotron wave generation during the approach phase of Rosetta to $67 \mathrm{P} / \mathrm{Churyumov-Gerasimenko} \mathrm{is} \mathrm{presented.} \mathrm{For} \mathrm{various} \mathrm{ac-}$ tivity levels of the comet, the crossing of the observational threshold is determined, whose level is derived from the wave power in the undisturbed solar wind near the comet's location during the approach phase at the appropriate frequency. The Giotto flyby at 27P/Grigg-Skjellerup is used to obtain an estimate of how often water-group ion cyclotron waves are observed, and to get insight into the wave forms. At 67P/Churyumov-Gerasimenko one can expect to observe water-group ion cyclotron waves already at a distance of $600000 \mathrm{~km}$ from the nucleus for a nominal outgassing rate of $Q=350 \times 10^{23}$ molecules per second. The observed first location of cyclotron waves during the Rosetta approach phase will give an indication of the actual outgassing rate of the comet.
\end{abstract}

Keywords. Interplanetary physics (MHD waves and turbulence) - space plasma physics (waves and instabilities)

\section{Introduction}

After its wake-up call on 20 January 2014, the Rosetta spacecraft (Glassmeier et al., 2007a) will fly to its rendezvous with comet 67P/Churyumov-Gerasimenko (CG), where it will arrive on 6 August 2014, when the approach phase switches to the surface mapping phase of the mission. At the time of the arrival, the actual gas production of the comet is only roughly known. The observations during the last years (e.g. (Schulz et al., 2004) and Weiler et al. (2004) or most recently Snodgrass et al. (2013)) predict at $3.5 \mathrm{AU}$ (20 August 2014) a total gas production rate of $2.1 \times 10^{25}$ (low-activity case, LAC), $3.5 \times 10^{25}$ (Snodgrass et al., 2013) or $3.8 \times 10^{26}$ molecules $\mathrm{s}^{-1}$ (high-activity case, HAC). However, the actual gas production rate is an important quantity in the mission planning. As reported by Snodgrass et al. (2013), the comet started its activity in the 2007/2008 at a heliocentric distance of $\sim 4.3 \mathrm{AU}$, and the authors predict a similar behaviour in 2014. Therefore, it is proposed to use the magnetometer of the Rosetta Plasma Consortium (RPC, Glassmeier et al., 2007b) to study to presence of ion cyclotron waves generated by freshly picked-up ions along the approach phase of Rosetta, concentrating on the last $2 \times 10^{6} \mathrm{~km}$. These measurements can provide an estimate for the gas production rate of CG early in the approach phase.

\section{Cometary outgassing and ion pickup}

As a comet moves towards the Sun and comes closer than the orbit of Jupiter, the solar radiation is strong enough to warm up the nucleus and to make it start outgassing. To describe the outgassing with a rate $Q$ [molecules s ${ }^{-1}$ ], a spherical expansion of the gas cloud at speed $V_{\mathrm{e}}$ is assumed, and an ionization rate $v\left[\mathrm{~s}^{-1}\right]$ is used for the loss through ionization. The number density $N_{\mathrm{c}}(r)$ of the gas cloud is then obtained as a function of radial distance $r$ from the nucleus, taking the loss through ionization into account:

$N_{\mathrm{c}}(r)=\frac{Q}{4 \pi V_{\mathrm{e}} r^{2}} \exp \left\{\frac{-v r}{V_{\mathrm{e}}}\right\}$.

Following Huddleston et al. (1990) all distances are scaled by $L=V_{\mathrm{e}} / \nu$, and the number of implanted ions per second into the solar wind is given by $N_{\mathrm{c}}(r) v$, giving rise to a total implanted particle flux $n_{\mathrm{i}} u_{\mathrm{i}}$ (with $n_{\mathrm{i}}$ and $u_{\mathrm{i}}$ the ion density and velocity respectively) at a specific location $x_{0}$ along the 
solar wind flow (taken to be along the $x$ direction):

$$
n_{\mathrm{i}} u_{\mathrm{i}}=\frac{Q}{4 \pi L^{2}} \int_{x_{0}}^{\infty} \frac{\exp \left\{-\left(x^{2}+y_{0}^{2}+z_{0}^{2}\right)\right\}}{x^{2}+y_{0}^{2}+z_{0}^{2}} \mathrm{~d} x .
$$

The mass loading by the picked-up ions will slow down the solar wind. In order to calculate this effect, Huddleston et al. (1990) calculated the solar wind speed from a solution of the one-dimensional magnetohydrodynamic (MHD) equations (see also Biermann et al., 1967) for a gas with two degrees of freedom $(f=2)$. However, Koenders et al. (2013) showed that the interaction of the outgassing comet with the incoming solar wind is better described by a gas with three degrees of freedom $(f=3)$. The continuity equations (see Koenders et al., 2013) for the flow have to be fulfilled:

$\frac{\partial}{\partial x}\left(n u_{x}\right)=M_{\mathrm{s}}$,

$\frac{\partial}{\partial x}\left(n u_{x}^{2}+p_{\mathrm{t}}\right)=I_{\mathrm{s}}$

$\frac{\partial}{\partial x}\left(\frac{1}{2} n u_{x}^{3}+\frac{(f+2)}{2} p_{\mathrm{t}} u_{\mathrm{x}}\right)=E_{\mathrm{s}}$,

where $n$ is the gas density, $u$ the gas flow velocity and $p_{\mathrm{t}}$ the thermal pressure. $M_{\mathrm{s}}, I_{\mathrm{s}}$ and $E_{\mathrm{s}}$ are the local density, momentum and energy sources. Biermann et al. (1967) showed that, of these, only $M_{\mathrm{S}}$ needs to be treated as non-zero, as long as the distance to the nucleus is much larger than stand-off distance of the ionopause of the comet. Therefore $I_{\mathrm{S}}=E_{\mathrm{s}}=0$ is assumed, and integrating Eqs. (4) and (5) from $-\infty$ to $x_{0}$, with the assumption that $p_{\infty}=0$ and some basic algebra, setting $f=3$ changes Eq. (13) in Huddleston et al. (1990)

$\frac{u_{\infty}}{u_{\mathrm{sw}}}=2-\sqrt{4-3\left(1+\frac{n_{\mathrm{i}} u_{\mathrm{i}} m_{i}}{n_{\mathrm{sw}} u_{\mathrm{sw}} m_{\mathrm{sw}}}\right)}$,

to this version:

$\frac{u_{\infty}}{u_{\mathrm{sw}}}=\frac{5}{2}-\sqrt{\left(\frac{5}{2}\right)^{2}-4\left(1+\frac{n_{\mathrm{i}} u_{\mathrm{i}} m_{i}}{n_{\mathrm{sw}} u_{\mathrm{sw}} m_{\mathrm{sw}}}\right)}$,

where $u_{\infty}$ is the undisturbed solar wind speed, $u_{\mathrm{sw}}$ the local (mass-loaded) solar wind speed and $n_{\text {sw }}$ the mass-loaded solar wind density, and the pickup flux $n_{\mathrm{i}} u_{\mathrm{i}}$ is obtained from Eq. (2).

From Eqs. (2) and (7) the pickup density and local (massloaded) solar wind speed can be obtained along the orbit of Rosetta, which is shown in Fig. 1 in cometocentric solar equatorial (CSEQ) coordinates, where the $x$ axis points towards the Sun, the $z$ axis is aligned with the solar rotational axis and the $y$ axis completes the triad.

The integral in Eq. (2) is numerically integrated, using the following parameters: $v=10^{-6} \mathrm{~s}^{-1}, V_{\mathrm{e}}=1 \mathrm{~km} \mathrm{~s}^{-1}$, and undisturbed solar wind conditions with $u_{\infty}=380 \mathrm{~km} \mathrm{~s}^{-1}$, $n_{\infty}=0.9 \times 10^{6} \mathrm{~m}^{-3}, B_{0}=1 \mathrm{nT}$ and the average solar wind ion mass $m_{\mathrm{sw}, \infty}=1.2 \mathrm{AMU}$.
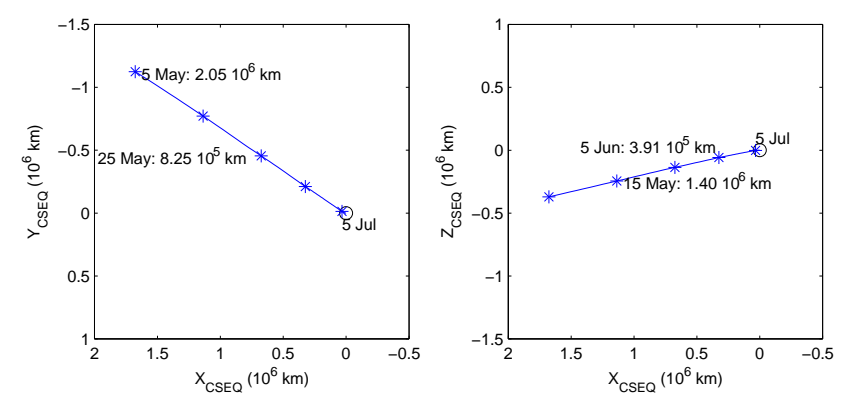

Fig. 1. The approach phase of Rosetta for the last $2 \times 10^{6} \mathrm{~km}$ in CSEQ coordinates. Asterisks indicate the respective date and radial distance from the nucleus.
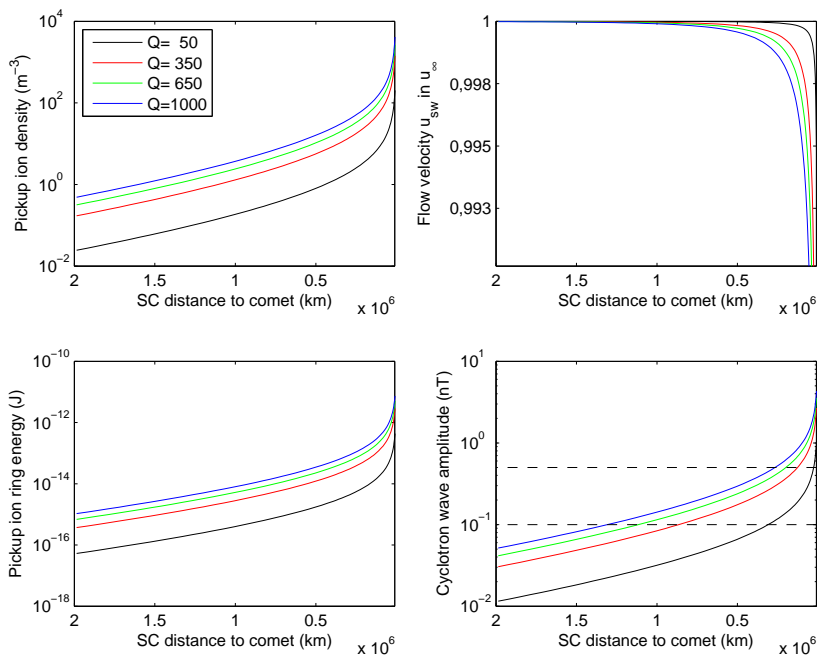

Fig. 2. Top left: the ion pickup density along the orbit of Rosetta for various outgassing rates 50 (black), 350 (red), 650 (green) and 1000 (blue) $10^{23} \mathrm{~s}^{-1}$. Top right: the mass-loaded flow velocity $u_{\mathrm{sw}}$ in units of $u_{\infty}$. Bottom left: the energy in the pickup ring distribution. Bottom right: the amplitude of the ion cyclotron waves assuming full scattering of the ring distribution. The dashed horizontal line at $0.1 \mathrm{nT}$ indicates the solar wind noise level. The upper dashed line marks the $0.5 \mathrm{nT}$ amplitude.

As the solar wind is not slowed significantly by the ion pickup during the approach phase, it is assumed under the square root of Eq. (7) that $u_{\mathrm{sw}}=u_{\infty}$ and $v_{\mathrm{sw}}=v_{\infty}$. The obtained $u_{\mathrm{sw}}$ at each point along the integration path is then used as $u_{\mathrm{i}}$ in the following step.

As the actual outgassing rate of $\mathrm{CG}$ is not known, a range of values for $Q$ has been used: 50, 350, 650 and $1000 \times 10^{23} \mathrm{~s}^{-1}$, where the middle values are compatible with the prediction model of Snodgrass et al. (2013) closer to the Sun. A nominal rate of $Q=350 \times 10^{23} \mathrm{~s}^{-1}$ will be used as a base and agrees reasonably with the estimate of Snodgrass et al. (2013) with CG near 3.5 AU during the approach phase. The results for the pickup density and the mass-loaded solar wind speed are shown in Fig. 2 top left and top right panels. 
In the assumption of a perpendicular magnetic field (i.e. $\boldsymbol{B}_{\mathrm{IMF}} \perp \boldsymbol{v}_{\mathrm{sw}}$ ), the freshly picked-up ions form a ring distribution, which is unstable for the generation of ion cyclotron (IC) waves (Lee, 1989; Gary, 1991) through scattering from a ring to a bi-spherical shell distribution (e.g. Huddleston and Johnstone, 1992). The total energy density in the ring distribution can be estimated as

$E_{\text {ring }}=0.5 n_{\mathrm{i}} m_{\mathrm{i}} u_{\mathrm{sw}}^{2}$,

where $m_{\mathrm{i}}$ is the ion mass, and the values for $n_{\mathrm{i}}$ and $u_{\mathrm{sw}}$ are taken from the calculation with Eq. (7) and shown in Fig. 2 top left and right panels. Assuming that all the energy of the ring distribution can be converted to IC wave energy, the amplitude of the waves can be estimated by

$B_{\text {wave }}=\sqrt{2 \mu_{0} E_{\text {ring }}}$.

The results of these two estimates are shown in Fig. 2 bottom left and right panels, respectively. The wave amplitude plot shows that for complete scattering and a moderate source rate of $Q=350 \times 10^{23} \mathrm{~s}^{-1}, 0.1 \mathrm{nT}$ waves may be expected already at $\sim 10^{6} \mathrm{~km}$ distance from the nucleus (lower dashed line). Unfortunately, the assumption of complete scattering is not valid and needs to be corrected.

Coates et al. (1989) defined an average age $\tau_{\mathrm{i}}$ of the implanted ions picked up upstream and seen at the spacecraft at location $x_{0}$ in the solar wind flow for an outgassing comet:

$\tau_{\mathrm{i}}=\frac{L}{u_{\mathrm{sw}}} \frac{\int_{x_{0}}^{\infty} \frac{\left(x-x_{0}\right) \exp \left\{-\left(x^{2}+y_{0}^{2}+z_{0}^{2}\right)^{1 / 2}\right\}}{x^{2}+y_{0}^{2}+z_{0}^{2}}}{\int_{x_{0}}^{\infty} \frac{\exp \left\{-\left(x^{2}+y_{0}^{2}+z_{0}^{2}\right)^{1 / 2}\right\}}{x^{2}+y_{0}^{2}+z_{0}^{2}}}$,

where, as above, the solar wind flow is assumed to be along the $x$ axis. This age of the ions should be compared to the scattering time $\tau_{\mathrm{s}}$ of the ions from a ring distribution to a shell distribution, which was found to be four times the gyroperiod in the wave field (Gaffey et al., 1988; Coates et al., 1989):

$\tau_{\mathrm{s}}=\frac{4}{\Omega_{\mathrm{i}}}\left(\frac{\Delta \boldsymbol{B}}{\boldsymbol{B}_{0}}\right)^{-1}$,

where $\Omega_{\mathrm{i}}$ is the gyro-frequency in the background field $\boldsymbol{B}_{0}$ and $\Delta \boldsymbol{B}$ is the wave field. Note that, because of the little pickup expected (because of the low outgassing rate) and thus the little slowdown of the solar wind, the interplanetary magnetic field field strength, $\boldsymbol{B}_{0}$, is kept constant in these calculations. It is clear from Fig. 3 top left panel that the age $\tau_{\mathrm{i}}$ of the implanted ions (thick magenta line) is much lower than the scattering time $\tau_{\mathrm{s}}$ (thin lines). This means that the ring distribution could have been fully scattered into a shell distribution, and thus the wave amplitude in Fig. 2 bottom right is an overestimation. In order to take into account the discrepancy between age and scatter time, the scattered energy is assumed to be

$E_{\text {scat }}=\frac{\tau_{\mathrm{i}}}{\tau_{\mathrm{s}}} E_{\text {ring }}$.
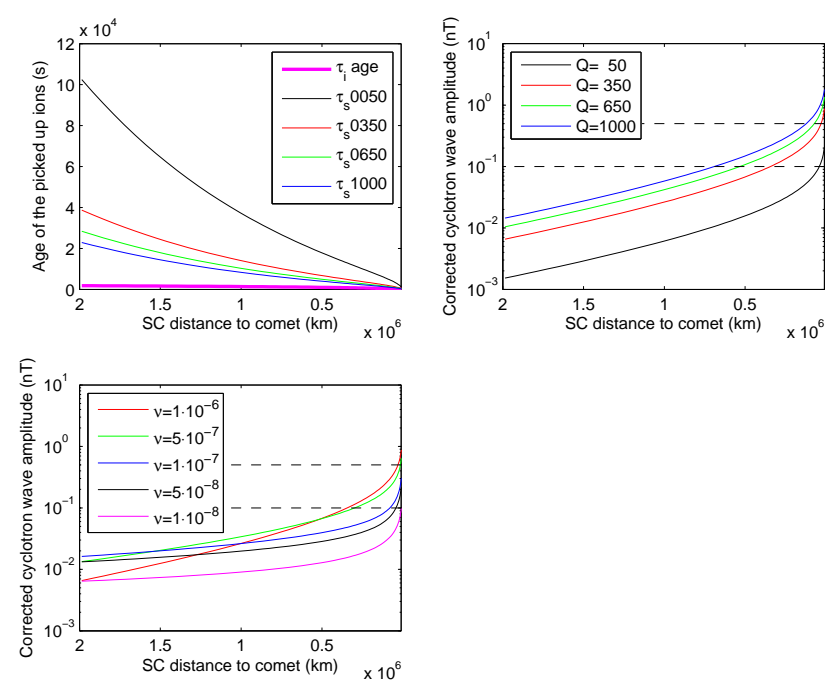

Fig. 3. Top left: the age $\tau_{\mathrm{i}}$ of the implanted ions and the scatter time $\tau_{\mathrm{s}}$ for different gas production rates. Top right: the corrected wave amplitude for different source rates. Bottom left: the scatter-timecorrected cyclotron wave amplitude for different values of the ionization rate $v$ and a constant outgassing rate of $Q=350 \times 10^{23} \mathrm{~s}^{-1}$. The lower horizontal dashed line marks $0.1 \mathrm{nT}$ solar wind noise level, and the upper dashed line shows the $0.5 \mathrm{nT}$ amplitude level.

The results of this correction are shown in Fig. 3 top right panel, where it is found that $0.1 \mathrm{nT}$ amplitude waves can be expected within the last $\sim 4 \times 10^{5} \mathrm{~km}$ from the nucleus for an outgassing rate of $Q=350 \times 10^{23} \mathrm{~s}^{-1}$.

All calculations, up to now, have assumed that the ionization rate is constant at $v=10^{-6} \mathrm{~s}^{-1}$. Figure 3 bottom left panel shows the corrected wave amplitude for an outgassing source rate of $Q=350 \times 10^{23} \mathrm{~s}^{-1}$ and an ionization rate $v$ varying between $10^{-6}$ and $10^{-8} \mathrm{~s}^{-1}$.

\section{Solar wind and Comet 27P/Grigg-Skjellerup}

The above theoretical calculations will probably contrast with the data from a real encounter with a comet. Therefore, the magnetometer data from the Giotto flyby of comet 27P/Grigg-Skjellerup (GS) are studied for IC waves (e.g. see Neubauer et al., 1993; Glassmeier and Neubauer, 1993; Coates et al., 1993). Naturally, there are great differences between the CG approach phase and the GS flyby: GS was much closer to the Sun, had a stronger outgassing rate of $Q=7 \times 10^{27} \mathrm{~s}^{-1}$ and had a fully developed bow shock. For CG a rate of $Q \leq 10^{26} \mathrm{~s}^{-1}$ is expected, and there will, most likely, only be a bow shock close to perihelion (Koenders et al., 2013). However, the situation upstream of a bow shock or Mach cone should not be affected by regions downstream. Thus in order to study the "homogeneity" of the ion pickup and IC wave generation in the upstream region of a comet, these data can be used. 

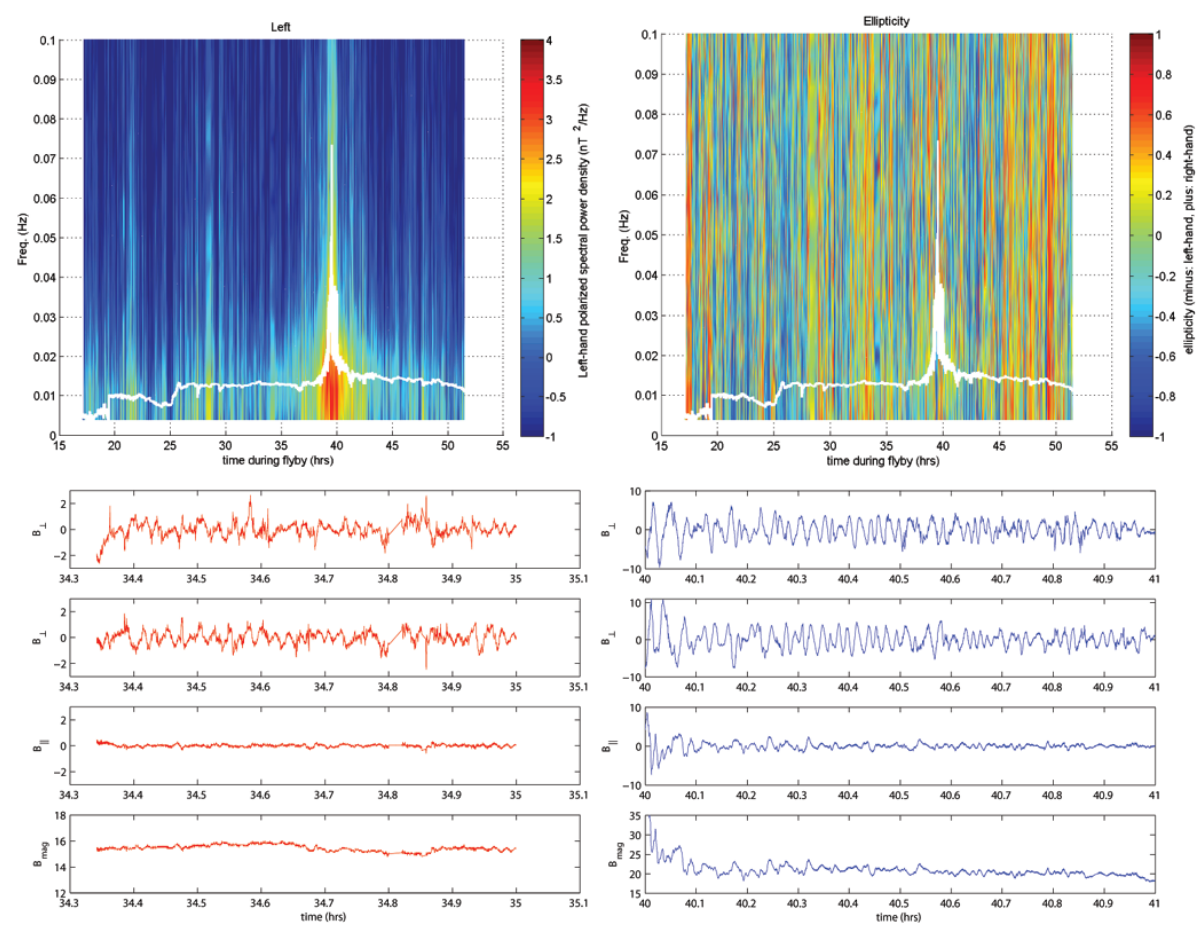

Fig. 4. Top left: the dynamic spectrum of the left-hand polarized magnetic field data from the Giotto magnetometer for the GS flyby from hour 15 to hour 56, where hour 0 is taken at 9 July 1992, DOY 191, at 00:00 UT. Closest approach was at hour 39.25. The white overlay line marks the $\mathrm{H}_{2} \mathrm{O}^{+}$cyclotron frequency. Top right: the dynamic ellipticity for the frequency estimates of the power spectrum in the left panel. Positive ellipticity for right-hand polarized, negative ellipticity for left-hand polarized waves. Bottom left: Giotto magnetometer data for hour 34-35 of the flyby, before closest approach, in MFA coordinates. The weak waves, at the water cyclotron frequency, are erratic and not well developed. Bottom right: Giotto magnetometer data for hour 40-41 of the flyby, after closest approach. The strong waves, at the water cyclotron frequency, are "anharmonic" but well developed.

The magnetometer data are transformed to a mean-fieldaligned (MFA) coordinate system, where the mean field is determined using a low-pass filter for periods above $30 \mathrm{~min}$, which represents the $Z_{\mathrm{MFA}}$ direction, and the $X_{\mathrm{MFA}}$ and $Y_{\mathrm{MFA}}$ directions are chosen perpendicular to this direction. The transverse components $X_{\mathrm{MFA}}$ and $Y_{\mathrm{MFA}}$ are combined into a left- and right-hand polarized components. Ion cyclotron waves are left-hand polarized waves at or below the local cyclotron frequency in the spacecraft frame (Gary, 1991). A dynamic spectrum is produced with spectral analysis (McPherron et al., 1972) on sliding windows of $256 \mathrm{~s}$ with a shift of $64 \mathrm{~s}$. The result for the left-hand polarized magnetic field component is shown in Fig. 4 top left panel, where the $x$ axis is time during the flyby, with closest approach at hour 39.25 , where hour 0 is taken at 9 July 1992 , DOY 191 , at 00:00 UT, the inbound bow shock crossing was at hour 36.8 and the outbound at hour 41.3. Figure 4 top right panel shows the ellipticity for the frequency estimates, where negative ellipticity means left-hand polarized waves. The white overplotted line in the panels marks the local $\mathrm{H}_{2} \mathrm{O}^{+}$cyclotron frequency.

The cyclotron waves are identified for frequencies $0.8-$ $1 f_{\mathrm{ci}}$, which have either a ratio of left-hand over right-hand power $R>1.5$ (e.g. see Delva et al., 2008) or an ellipticity $E<0$. In the interval shown in Fig. 4 and Giotto within $500000 \mathrm{~km}$ from the nucleus, there are a total of $1096 \mathrm{spec}-$ tra of which 441 have $R>1.5$ and 474 have $E<0$, with the stronger restriction $R$ contained in the weaker $E$. This means that only $\sim 40 \%$ of the time during the flyby, IC waves were observed at GS. Two examples of IC wave intervals are shown in Fig. 4: bottom left the data for hour 3435 (before closest approach and upstream of the bow shock), where there are irregular waves at the cyclotron frequency ( $f_{\mathrm{ci}} \approx 13 \mathrm{mHz}$ ); bottom right the data for hour $40-41$ (after closest approach and downstream of the bow shock), where the waves look "anharmonic" (Glassmeier et al., 1997) at the cyclotron frequency $\left(f_{\mathrm{ci}} \approx 17 \mathrm{mHz}\right.$ ), but they are much better developed than in the earlier interval. Interestingly, calculating the ratio of the average age of the implanted ions and the scattering time reveals values of 4.3 and 4.5 for both intervals respectively. This means that, although the waves have had ample time to grow in both intervals, only the post-closest approach interval has well-developed, albeit "anharmonic" waves. This could indicate a difference in wave growth in the unshocked (first interval) and shocked solar wind plasma (second interval). 

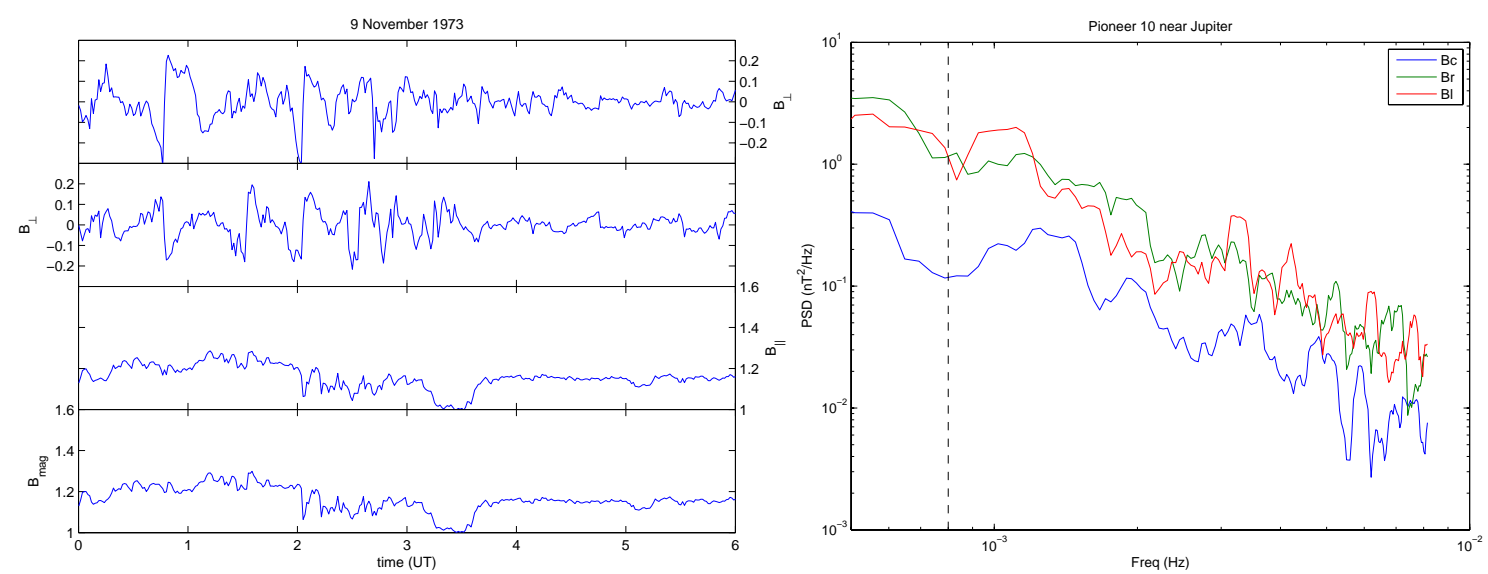

Fig. 5. Left: Pioneer 10 magnetometer data for 9 November 1973, 00:00-06:00 UT near Jupiter orbit, in MFA coordinates. Right: power spectra of the data from the compressional, left- and right-hand polarized components. The vertical dashed line shows the $\mathrm{H}_{2} \mathrm{O}^{+}$cyclotron frequency for this interval.

In order to find the minimal power level that the cyclotron waves need to achieve to be visible over the solar wind background noise, the magnetometer data from Pioneer 10 during its approach of Jupiter are studied at a radial distance from the Sun comparable to Rosetta's approach to CG. The data and the power spectrum are shown in Fig. 5. At the local IC frequency $\left(f_{\mathrm{ci}} \approx 0.8 \mathrm{mHz}\right)$, the wave power is $\sim 1.9 \mathrm{nT}^{2} \mathrm{~Hz}^{-1}$, which corresponds to a wave amplitude of $0.1 \mathrm{nT}$. This gives an indication of the spectral power density that might be expected during the approach phase and of the cyclotron wave amplitudes that can be observed unambiguously by the Rosetta Plasma Consortium instruments.

\section{Expectations for the approach phase}

A theoretical model for the IC wave production along the approach of Rosetta to CG has been presented in order to make an early estimate of the activity level of the comet from the magnetometer data. In combination with data from the Giotto flyby of GS and solar wind data from Pioneer 10 near Jupiter, the following conclusions can be made:

- the solar wind noise level at the water cyclotron frequency is $\sim 0.1 \mathrm{nT}$, which sets a lower limit to the amplitude of the observable IC waves;

- depending on the outgassing rate, the waves cross the $0.1 \mathrm{nT}$ threshold between 6 and $3.5 \times 10^{5} \mathrm{~km}$ distance from CG for a source rate $1000 \times 10^{23} \geq Q \geq 350 \times$ $10^{23} \mathrm{~s}^{-1}$;

- lower outgassing rates than the nominal value used in this paper will further reduce the radial distance at which IC waves may be observable;

- the spacecraft approach from $6 \times 10^{5} \mathrm{~km}$ to closest distance takes place during 24 days of the mission. Based on observations at GS, IC waves are expected to be observable $40 \%$ of the time, which would give an accumulated 10 days of IC wave observation;

- because of the young age of the ions, the wave form might not be well developed;

- shocked and unshocked solar wind may have influence on wave growth and form; however, Rosetta will most likely not encounter a bow shock during its approach phase.

Although in the beginning of the outgassing of CG the sublimation of $\mathrm{CO}_{2}$ will dominate that of $\mathrm{H}_{2} \mathrm{O}$ by a factor $\sim 5$ (see Snodgrass et al., 2013, Table 6), this paper concentrated on water group ions because of the much heavier molecular mass of carbon dioxide (44 vs. 18 AMU). The heavier mass lowers the cyclotron frequency by a factor $2.5: f_{\mathrm{H}_{2} \mathrm{O}} \approx 0.85$ and $f_{\mathrm{CO}_{2}} \approx 0.35 \mathrm{mHz}$, which makes the heavier ions more difficult to detect at low amplitude because of the long time segments of relatively constant solar wind conditions needed (e.g. see Delva et al., 2011, for a discussion of IC waves at Venus for varying solar wind cone angle).

In the middle of 2014, when the magnetometer data from the Rosetta approach phase will have arrived on Earth, an actual estimate of the outgassing rate of the comet can be made from the $\mathrm{H}_{2} \mathrm{O}^{+}$IC wave observations.

Acknowledgements. The authors would like to acknowledge the PDS/PPI for the Giotto and Pioneer 10 magnetometer data. The work of K.-H. Glassmeier, I. Richter and C. Koenders was financially supported by the German Bundesministerium für Wirtschaft und Technologie and the Deutsches Zentrum für Luft- und Raumfahrt under contract 50 QP 1001 for Rosetta.

Topical Editor L. Blomberg thanks one anonymous referee for his/her help in evaluating this paper. 


\section{References}

Biermann, L., Brosowski, B., and Schmid, H. U.: The interactions of the solar wind with a comet, Sol. Phys., 1, 254-284, doi:10.1007/BF00150860, 1967.

Coates, A. J., Johnstone, A. D., Wilken, B., Jockers, K., and Glassmeier, K.-H.: Velocity space diffusion of pickup ions from the water group at comet Halley, J. Geophys. Res., 94, 9983-9993, 1989.

Coates, A. J., Johnstone, A. D., Huddleston, D. E., Wilken, B., Jockers, K., Borg, H., Amata, E., Formisano, V., Bavassano-Cattaneo, M. V., Winningham, J. D., Gorgiolo, C., and Neubauer, F. M.: Pickup water group ions at comet Grigg-Skjellerup, Geophys. Res. Lett., 20, 483-486, 1993.

Delva, M., Zhang, T. L., Volwerk, M., Vörös, Z., and Pope, S. A.: Proton cyclotron waves in the solar wind at Venus, Geophys. Res. Lett., 113, E00B06, doi:10.1029/2008JE003148, 2008.

Delva, M., Mazelle, C., Bertucci, C., Volwerk, M., Vörös, Z., and Zhang, T. L.: Proton cyclotron wave generation mechanisms upstream of Venus, J. Geophys. Res., 116, A02318, doi:10.1029/2010JA015826, 2011.

Gaffey, J. D., Winske, D., and Wu, C. S.: Time scales for formation and spreading of velocity shells of pickup ions in the solar wind, J. Geophys. Res., 93, 5470-5486, 1988.

Gary, S. P.: Electromagnetic ion/ion instabilities and their consequences in space plasmas: A review, Space Sci. Rev., 56, 373415, 1991.

Glassmeier, K.-H. and Neubauer, F. M.: Low-frequency electromagnetic plasma waves at comet P/Grigg-Skjellerup: overview and spectral characteristics, J. Geophys. Res., 98, 20921-20935, 1993.

Glassmeier, K.-H., Tsurutani, B. T., and Neubauer, F. M.: Adventures in parameter space: a comparison of low-frequency plasma waves at comets, in: Nonlinear waves and chaos in space plasmas, edited by: Hada, T. and Matsumoto, H., 77-119, Terrapub, Germany, 1997.

Glassmeier, K.-H., Boehnhardt, H., Koschny, D., Kührt, E., and Richter, I.: The Rosetta mission: flying towards the origin of the solar system, Space Sci. Rev., 128, 1-21, 2007a.
Glassmeier, K.-H., Richter, I., Diedrich, A., Musmann, G., Auster, U., Motschmann, U., Balogh, A., Carr, C., Cupido, E., Coates, A., Rother, M., Schwingenschuh, K., Szegö, K., and Tsurutani, B.: RPC-MAG the flux-gate magnetometer in the ROSETTA plasma consortium, Space Sci. Rev., 128, 649-670, 2007b.

Huddleston, D. E. and Johnstone, A. D.: Relationship between wave energy and free energy from pickup ions in the comet Halley environment, J. Geophys. Res., 97, 12217-12230, 1992.

Huddleston, D. E., Johnstone, A. D., and Coates, A. J.: Determination of comet Halley gas emission characteristics from mass loading of the solar wind, J. Geophys. Res., 95, 21-30, 1990.

Koenders, C., Glassmeier, K.-H., Richter, I., Motschmann, U., and Rubin, M.: Revisiting cometary bow shock positions, Planet. Space Sci., 87, 85-95, doi:10.1016/j.pss.2013.08.009, 2013.

Lee, M. A.: Plasma waves and instabilities at comets and in magnetospheres, AGU monograph 53, in: Ultra-low frequency waves at comets, edited by: Tsurutani, B. T. and Oya, H., 13-29, AGU, Washington, DC, USA, 1989.

McPherron, R. L., Russell, C. T., and Coleman, P. J.: Fluctuating magnetic fields in the magnetosphere, II., ULF waves, Space Sci. Rev., 13, 411-454, 1972.

Neubauer, F. M., Marschall, H., Pohl, M., Glassmeier, K.-H., Musmann, G., Mariani, F., Acuna, H. G., Burlaga, L. F., Ness, N. F., Wallis, M. K., Schmidt, H. U., and Ungstrup, E.: First results from the Giotto magnetometer experiment during the P/GriggSkjellerup encounter, Astron. Astrophys., 268, L5-L8, 1993.

Schulz, R., Stüwe, J. A., and Boehnhardt, H.: Rosetta target comet 67P/Churyumov-Gerasimenko: Postperihelion gas and dust production rates, Astron. Astrophys., 422, L19-L21, 2004.

Snodgrass, C., Tubiana, C., Bramich, D. N., Meech, K., Boehnhardt, H., and Barrera, L.: Beginning of activity in 67P/Churyumov-Gerasimenko and predictions for 20142015, Astron. Astrophys., 557, 15 pp., doi:10.1051/00046361/201322020, 2013.

Weiler, M., Rauer, H., and Helbert, J.: Optical observations of Comet 67P/Churyumov-Gerasimenko, Astron. Astrophys., 414, 749-755, 2004. 